\title{
Reconstruction of Discrete Sets with Absorption
}

\author{
Attila Kuba ${ }^{1}$ and Maurice Nivat ${ }^{2}$ \\ 1 Department of Applied Informatics, University of Szeged, \\ Árpád tér 2, H-6720 Szeged, Hungary \\ kuba@inf.u-szeged.hu \\ 2 Laboratoire d'Informatique Algorithmique: Fondements et Applications, \\ Université Paris 7 Denis-Diderot, Paris, France \\ tcsmn@club-internet.fr
}

\begin{abstract}
A generalization of a classical discrete tomography problem is considered: Reconstruct binary matrices from their absorbed row and columns sums, i.e., when some known absorption is supposed. It is mathematically interesting when the absorbed projection of a matrix element is the same as the absorbed projection of the next two consecutive elements together. We show that, in this special case, the non-uniquely determined matrices contain a certain configuration of $0 \mathrm{~s}$ and $1 \mathrm{~s}$, called alternatively corner-connected components. Furthermore, such matrices can be transformed into each other by switchings the 0s and $1 \mathrm{~s}$ of these components.
\end{abstract}

\section{Introduction}

Consider a ray (such as light or X-ray) transmitting through homogeneous material. It is a well-known physical phenomenon that a part of the ray will be absorbed in the material. Quantitatively, let $I_{0}$ and $I$ denote the initial and the detected intensities of the ray. Then

$$
I=I_{0} \cdot e^{-\mu x}
$$

where $\mu \geq 0$ denotes the absorption coefficient of the material and $x$ is the length of the path of the ray in the material.

Consider now the 2-dimensional integer lattice $\mathbb{Z}^{2}$. Let $m$ and $n$ be positive integers. A discrete rectangle with size $m \times n$ is a special discrete set of $\mathbb{Z}^{2}$ determined as the intersection of $m$ consecutive horizontal lattice lines with $n$ consecutive vertical lattice lines. If $F$ is a discrete set in $\mathbb{Z}^{2}$, then there is an $m \times n$ discrete rectangle containing $F$, it is called containing (discrete) rectangle. (For the sake of simplicity we take the smallest containing rectangle in the following.)

Let us suppose that the horizontal and vertical projections of $F$ are measured by detectors placed in the next column to left and in the next row to above, respectively, of the containing rectangle (see Fig. 1.a).

Then the projections can be computed according to (1). For example, in the case of the discrete set $F$ given in Fig. 1a, the projections along the horizontal 


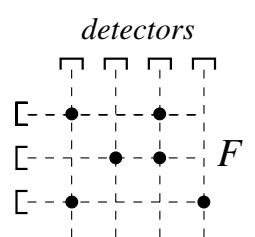

a)

$$
\begin{array}{|l|l|l|l|l|l} 
& s_{1} & s_{2} & s_{3} & s_{4} \\
\hline r_{1} & 1 & 0 & 1 & 0 \\
\hdashline r_{2} & 0 & 1 & 1 & 0 & A \\
\hdashline r_{3} & 1 & 0 & 0 & 1
\end{array}
$$

b)

Fig. 1. A 2D discrete set $F$, the corresponding binary matrix $A$, and the horizontal and vertical projections. (a) A $2 \mathrm{D}$ discrete set $F$ (its elements are indicated by points) and the detectors measuring its horizontal and vertical projections. (b) The binary matrix $A$ and its row and column sums

lattice lines of the containing rectangle are $r_{1}=e^{-\mu \cdot 1}+e^{-\mu \cdot 3}, r_{2}=e^{-\mu \cdot 2}+e^{-\mu \cdot 3}$, and $r_{3}=e^{-\mu \cdot 1}+e^{-\mu \cdot 4}$ (from top to down).

Let us use an equivalent representation of the $2 \mathrm{D}$ discrete sets and their horizontal and vertical projections. The containing rectangle including $F$ can be represented by a binary matrix $A=\left(a_{i j}\right)_{m \times n}$ as follows (see Fig. 1.b): $a_{i j}=1$ if the lattice point corresponding to $(i, j)$ is an element of $F, a_{i j}=0$ otherwise. In order to use the generally accepted notation of numeration systems [5] let us introduce

$$
\beta=e^{\mu}
$$

Clearly, $\beta \geq 1$, because $\mu>0$. Then we can define the absorbed row and column sums of $A, R_{\beta}(A)$ and $S_{\beta}(A)$, respectively, as

$$
R_{\beta}(A)=\left(r_{1}, \ldots, r_{m}\right), \quad \text { where } \quad r_{i}=\sum_{j=1}^{n} a_{i j} \beta^{-j}, \quad i=1, \ldots, m
$$

and

$$
S_{\beta}(A)=\left(s_{1}, \ldots, s_{n}\right), \quad \text { where } \quad s_{j}=\sum_{i=1}^{m} a_{i j} \beta^{-i}, \quad j=1, \ldots, n .
$$

For example, in the case of matrix $A$ given in Fig. 1.b) $R_{\beta}(A)=\left(\beta^{-1}+\right.$ $\left.\beta^{-3}, \beta^{-2}+\beta^{-3}, \beta^{-1}+\beta^{-4}\right)$ and $S_{\beta}(A)=\left(\beta^{-1}+\beta^{-3}, \beta^{-2}, \beta^{-1}+\beta^{-2}, \beta^{-3}\right)$.

Then the reconstruction problem of binary matrices with absorption knowing the projections along horizontal and vertical lines can be posed as 
ReCOnStRUCTION $D A 2 D(\beta)$.

Given: $\quad \beta \geq 1, m, n, R \in \mathbb{N}^{m}$, and $S \in \mathbb{N}^{n}$.

Task: $\quad$ Construct a binary matrix $A$ with size $m \times n$ such that

$$
R_{\beta}(A)=R \quad \text { and } \quad S_{\beta}(A)=S .
$$

We say that a binary matrix $A$ is a solution of the $D A 2 D(\beta)$ reconstruction problem if (5) is satisfied.

If $\beta=1$ then we have the classical reconstruction problem of binary matrices without absorption (as summaries see e.g. [1,3]). Select, now, a mathematically interesting case when $\beta=\beta_{0}$ where

$$
\beta_{0}^{-1}=\beta_{0}^{-2}+\beta_{0}^{-3}
$$

giving

$$
\beta_{0}=\frac{1+\sqrt{5}}{2} .
$$

(The other solution of $(6)$, namely $(1-\sqrt{5}) / 2$, is not applicable in this case, see (2).)

In this paper we discuss the problem of reconstruction of binary matrices from their row and column sums in the case of absorption corresponding to $\beta_{0}$. Because of the page limit, the proofs of theorems and lemmas are omitted here (but they are given in [4]).

\section{$2 \quad \boldsymbol{\beta}_{0}$-Representation}

Consider the absorbed row and column sums of the binary matrix $A$ in the case of $\beta=\beta_{0}$ :

$$
r_{i}=\sum_{j=1}^{n} a_{i j} \beta_{0}^{-j}, i=1, \ldots, m, \quad \text { and } \quad s_{j}=\sum_{i=1}^{m} a_{i j} \beta_{0}^{-i}, j=1, \ldots, n \text {. }
$$

Using the terminology of numeration systems we can say that the finite (binary) word $a_{i 1} \cdots a_{i n}$ is a (finite) representation in base $\beta_{0}$ (or a finite $\beta_{0}$ representation) of $r_{i}$ for each $i=1, \ldots, m$, and, similarly, $a_{1 j} \cdots a_{m j}$ is a $\beta_{0^{-}}$ representation of $s_{j}$ for each $j=1, \ldots, n$. The equations (8) mean also that the row and column sums of $A$ are nonnegative real numbers having a finite $\beta_{0^{-}}$ representation with $n$ and $m$ binary digits, respectively (including the eventually ending zeros). 
Let $B_{k}$ denote the set of nonnegative real numbers having a $\beta_{0}$-representation with $k$ binary digits $(k>1)$, formally,

$$
B_{k}=\left\{\sum_{i=1}^{k} a_{i} \beta_{0}^{-i} \mid a_{i} \in\{0,1\}\right\}
$$

Then

$$
r_{i} \in B_{n}, i=1, \ldots, m, \quad \text { and } \quad s_{j} \in B_{m}, j=1, \ldots, n,
$$

are necessary conditions for the existence of a matrix $A$ with

$$
R_{\beta_{0}}(A)=\left(r_{1}, \ldots, r_{m}\right) \quad \text { and } \quad S_{\beta_{0}}(A)=\left(s_{1}, \ldots, s_{n}\right)
$$

\subsection{Switching in $\boldsymbol{\beta}_{0}$-Representations}

The $\beta_{0}$-representation is generally non-unique, because there are binary words with the same length representing the same number. For example, on the base of (6) it is easy to check the following equality between the 3 -digit-length $\beta_{0^{-}}$ representations

$$
100=011
$$

Furthermore, Equality (12) may allow us to generate newer $\beta_{0}$-representations: If in a finite length $\beta_{0}$-representation there is one of the sub-words 100 or 011 , then it can be replaced by the other one. This transformation of binary words is called (1D) elementary switching.

It is clear that one or more $1 \mathrm{D}$ elementary switchings give(s) new $\beta_{0}$-representation(s) of the same number. As an example, see the 5 -digit-length $\beta_{0}$-representations of $1 / \beta_{0}$ :

$$
10000=01100=01011
$$

where the second $\beta_{0}$-representation is created from the first representation by switching on the first three positions and the third representation is created from the second one by switching on the last three positions.

As direct consequences of (12), it is easy to see that

$$
\begin{aligned}
10 x 00 & =01 x 11 \\
10 x 0 x 00 & =01 x 1 x 11 \\
10 x 0 x 0 x 00 & =01 x 1 x 1 x 11 \\
& \ldots
\end{aligned}
$$


where $x$ denotes the positions where both $\beta_{0}$-representations have the same binary digit. (That is, such kind of transformation $1(0 x)^{k} 00 \leftrightarrow 0(1 x)^{k} 11(k \geq$ 0 ) between the sub-words of the $\beta_{0}$-representations can be performed without changing the represented value and without changing the values in the positions indicated by $x$.) The transformations described by (12) and (14) are called (1D) switchings.

Now, we are going to prove that any finite $\beta_{0}$-representation of a number can be get from its any other $\beta_{0}$-representation by switchings.

Lemma 1. Let $a_{1} \cdots a_{k}$ and $b_{1} \cdots b_{k}$ be different, $k$-digit-length $\beta_{0}$-representations of the same number. Then $b_{1} \cdots b_{k}$ can be get from $a_{1} \cdots a_{k}$ by a finite number of $1 D$ switchings.

Proof. A procedure by which the suitable switchings can be found is described in [4].

Consequence. If $a_{1} \cdots a_{k}$ and $b_{1} \cdots b_{k}$ are different, $k$-digit-length $\beta_{0}$-representations of the same number, then there are positions $i, i+1, i+2(1 \leq i \leq k-2)$ such that there is a switching between $a_{1} \cdots a_{k}$ and $b_{1} \cdots b_{k}$ on these positions.

\section{2D Switchings}

In this section we determine the 2D switchings, i.e., the transformations of binary matrices by which some of their 0 s and 1 s can be switched to each other such that the absorbed row and column sums remain the same.

\subsection{Connectedness}

Consider the class of $m \times n(m, n \geq 3)$ binary matrices with given row and column sum vectors in the case of absorption corresponding to $\beta_{0}$. Let $S_{(i, j)}$ $(1<i<m, 1<j<n)$ denote the $3 \times 3$ discrete square

$$
S_{(i, j)}=\{i-1, i, i+1\} \times\{j-1, j, j+1\} .
$$

Let $\Sigma$ be a set of $3 \times 3$ discrete squares of $\{1, \ldots, m\} \times\{1, \ldots, n\}$ and let $S_{(i, j)}, S_{\left(i^{\prime}, j^{\prime}\right)} \in \Sigma$. Two kinds of connectedness will be defined on the set of $3 \times 3$ discrete squares: side-connectedness and corner-connectedness. There is sideconnection between $S_{(i, j)}$ and $S_{\left(i^{\prime}, j^{\prime}\right)}$ if $\left(i^{\prime}, j^{\prime}\right) \in\{(i-2, j),(i, j-2),(i, j+2),(i+$ $2, j)\}$. The squares being side-connected to $S_{(i, j)}$ are called the side-neighbors of $S_{(i, j)}$. As an illustration see Fig. 2.a. The squares $S_{(i, j)}$ and $S_{\left(i^{\prime}, j^{\prime}\right)}$ are cornerconnected if $\left(i^{\prime}, j^{\prime}\right) \in\{(i-2, j-2),(i-2, j+2),(i+2, j-2),(i+2, j+2)\}$. The squares being corner-connected to $S_{(i, j)}$ are called the corner-neighbors of $S_{(i, j)}$ (see Fig. 2.b).

There is a side-chain between $S_{(i, j)}$ and $S_{\left(i^{\prime}, j^{\prime}\right)}$ in $\Sigma$ if a sequence of elements of $\Sigma$ can be selected such that the first element is $S_{(i, j)}$ and the last element is $S_{\left(i^{\prime}, j^{\prime}\right)}$ and any two consecutive elements of the sequence are side-connected. (A sequence consisting of only one square is a side-chain by definition.) 


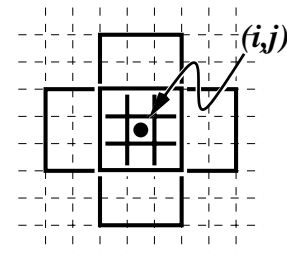

a)

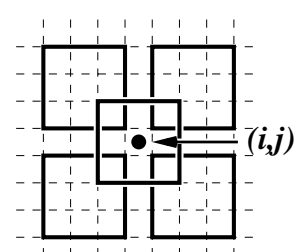

b)

Fig. 2. The discrete square $S_{(i, j)}$ and a) its side-neighbors and b) its cornerneighbors

The set $\Sigma$ is side-connected if there is a side-chain in $\Sigma$ between its any two different elements (see Fig. 3.a). A side-connected set $\Sigma$ is strongly sideconnected if whenever $S_{(i, j)}, S_{\left(i^{\prime}, j^{\prime}\right)} \in \Sigma$ and $S_{(i, j)} \cap S_{\left(i^{\prime}, j^{\prime}\right)} \neq \emptyset$ then they are side-connected or they have a common side-connected neighbor (see Fig. 3.b). A maximal strongly side-connected subset of $\Sigma$ is called a strongly side-connected component of $\Sigma$.

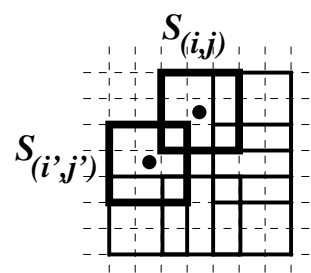

a)

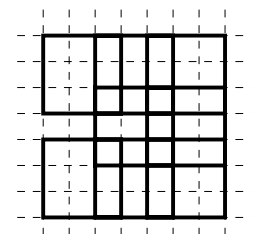

b)

Fig. 3. Side-connected sets of discrete squares. The set a) is not strongly side connected because $S_{(i, j)} \cap S_{\left(i^{\prime}, j^{\prime}\right)} \neq \emptyset$ but they are not side-connected and they have no common side-neighbor. b) A strongly side-connected set

Let $\sigma$ be a set of strongly side-connected components $\Sigma^{(1)}, \ldots, \Sigma^{(k)}(k \geq 1)$. Let $\Sigma^{(l)}, \Sigma^{\left(l^{\prime}\right)} \in \sigma\left(1 \leq l, l^{\prime} \leq k\right) . \Sigma^{(l)}$ and $\Sigma^{\left(l^{\prime}\right)}$ are corner-connected if whenever $S \in \Sigma^{(l)}$ and $S^{\prime} \in \Sigma^{\left(l^{\prime}\right)}$ have a common position then $S$ and $S^{\prime}$ are cornerconnected squares. (Since $\Sigma^{(l)}$ and $\Sigma^{\left(l^{\prime}\right)}$ are maximal, $S$ and $S^{\prime}$ cannot be sideconnected squares.) There is a corner-chain between $\Sigma^{(l)}$ and $\Sigma^{\left(l^{\prime}\right)}$ in $\sigma$ if a sequence of elements of $\sigma$ can be selected such that the first element is $\Sigma^{(l)}$ and the last element is $\Sigma^{\left(l^{\prime}\right)}$ and any two consecutive elements of the sequence are corner-connected. (A sequence consisting of only one component is a cornerchain by definition.) The set $\sigma$ is corner-connected if there is a corner-chain in $\sigma$ between its any two elements (see Fig. 4.a). 


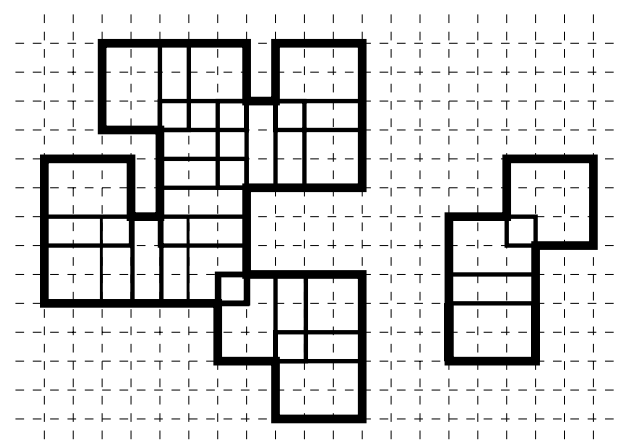

Fig. 4. Two corner-connected sets, both of them have two strongly sideconnected components

\subsection{Switching Patterns}

In order to identify not necessarily rectangular parts of binary matrices, we introduce the concept of binary patterns (or shortly, patterns) as binary valued functions defined on an arbitrary non-empty subset of $\{1, \ldots, m\} \times\{1, \ldots, n\}$. (In this terminology binary matrices are binary patterns on discrete rectangles.)

Let $P$ be a binary pattern, its domain will be denoted by $\operatorname{dom}(P)$. The absorbed row and column sums of $P$ are denoted by $R_{\beta_{0}}(P)$ and $S_{\beta_{0}}(P)$, respectively, where the $i$ th component of $R_{\beta_{0}}(P)$ is

$$
\sum_{(i, j) \in \operatorname{dom}(P)} P(i, j) \cdot \beta_{0}^{-j}
$$

for $1 \leq i \leq m$ and the $j$ th component of $S_{\beta_{0}}(P)$ is

$$
\sum_{(i, j) \in \operatorname{dom}(P)} P(i, j) \cdot \beta_{0}^{-i}
$$

for $1 \leq j \leq n$.

Let us define the switching pair of $P, P^{\prime}$, as

$$
P^{\prime}(i, j)=1-P(i, j)
$$

on $\operatorname{dom}(P)$. We say that $P$ is a switching pattern if

$$
R_{\beta_{0}}(P)=R_{\beta_{0}}\left(P^{\prime}\right) \quad \text { and } \quad S_{\beta_{0}}(P)=S_{\beta_{0}}\left(P^{\prime}\right),
$$

and $P$ and $P^{\prime}$ are a switching pair. That is, the patterns of a switching pair can be get from each other by switching their 0 s and 1 s and still they have the same absorbed row and column sums. 
Consider the following binary patterns:

$$
E_{(i, j)}^{(0)}=\begin{array}{lll}
0 & 1 & 1 \\
1 & 0 & 0 \\
1 & 0 & 0
\end{array} \quad \text { and } E_{(i, j)}^{(1)}=\begin{array}{lll}
1 & 0 & 0 \\
0 & 1 & 1 \\
0 & 1 & 1
\end{array},
$$

both of them are defined on the discrete square $S_{(i, j)}$. It is easy to check that $E_{(i, j)}^{(0)}$ and $E_{(i, j)}^{(1)}$ are a switching pair. They play an important role in the generation of switching patterns, $E_{(i, j)}^{(0)}$ and $E_{(i, j)}^{(1)}$ are called the 0-type and 1-type elementary (2D) switching pattern, respectively.

\subsection{Composition of Patterns}

The composition of two patterns $P$ and $P^{\prime}$ is the function

$$
P * P^{\prime}: \operatorname{dom}(\mathrm{P}) \triangle \operatorname{dom}\left(\mathrm{P}^{\prime}\right) \longrightarrow\{0,1\}
$$

$(\triangle$ denotes the symmetric difference) such that

$$
\left[P * P^{\prime}\right](i, j)= \begin{cases}P(i, j), & \text { if }(i, j) \in \operatorname{dom}(P) \backslash \operatorname{dom}\left(P^{\prime}\right), \\ P^{\prime}(i, j), & \text { if }(i, j) \in \operatorname{dom}\left(P^{\prime}\right) \backslash \operatorname{dom}(P) .\end{cases}
$$

(That is, $P * P^{\prime}$ is undefined on $\operatorname{dom}(P) \cap \operatorname{dom}\left(P^{\prime}\right)$.)

For example,

$$
\begin{aligned}
& 011 \\
& E_{(i, j)}^{(0)} * E_{(i+2, j)}^{(0)}=\begin{array}{rll}
1 & 0 & 0 \\
x & x & x \\
1 & 0 & 0
\end{array},
\end{aligned}
$$

defined on $S_{(i, j)} \triangle S_{(i+2, j)}=\{i-1, i, i+2, i+3\} \times\{j-1, j, j+1\}$. (Just for the sake of simple presentation, on the right side of (15) the whole sub-matrix on the rectangle $\{i-1, \ldots, i+3\} \times\{j-1, j, j+1\}$ is indicated and $x$ denotes the positions in the sub-matrix where the composition is undefined.) Similarly,

$$
\begin{aligned}
& 01 x 11
\end{aligned}
$$

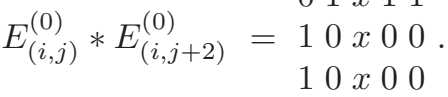

defined on $S_{(i, j)} \triangle S_{(i, j+2)}=\{i-1, i, i+1\} \times\{j-1, j, j+2, j+3\}$. It is easy to see that $E_{(i, j)}^{(0)} * E_{(i+2, j)}^{(0)}$ and $E_{(i, j)}^{(0)} * E_{(i, j+2)}^{(0)}$ are switching patterns, their switching 
pairs are $E_{(i, j)}^{(1)} * E_{(i+2, j)}^{(1)}$ and $E_{(i, j)}^{(1)} * E_{(i, j+2)}^{(1)}$, respectively, where

$$
E_{(i, j)}^{(1)} * E_{(i+2, j)}^{(1)}=\begin{array}{llll}
1 & 0 & 0 \\
0 & 1 & 1 \\
& x & x & x \\
0 & 1 & 1 \\
0 & 1 & 1
\end{array} \quad \text { and } \quad E_{(i, j)}^{(1)} * E_{(i, j+2)}^{(1)}=\begin{array}{lllll}
1 & 0 & x & 0 & 0 \\
0 & 1 & x & 1 & 1 \\
0 & 1 & x & 1 & 1
\end{array} .
$$

These examples show how new switching patterns can be created from elementary switching patterns by composition. Now, we show a general way how two (not only elementary) switching patterns can be used to generate another switching pattern.

\subsection{Composition of Elementary Switching Patterns}

Let $\mathcal{E}=\left\{E_{1}, \ldots, E_{k}\right\}(k \geq 1)$ be a set of elementary switching patterns of the same type on a strongly side-connected set $\Sigma=\left\{S_{1}, \ldots, S_{k}\right\}$. The composition of $\mathcal{E}$ on $\Sigma$, denoted by $\mathcal{C}(\mathcal{E})$, is defined as follows. If $k=1$ then $\mathcal{C}(\mathcal{E})=E_{1}$. If $k>1$ then let us suppose that $S_{1}, \ldots, S_{k}$ are indexed such that for each $l(<$ $k)\left\{S_{1}, \ldots, S_{l}\right\}$ is a strongly side-connected set and one of its squares is sideconnected with $S_{l+1}$. (It is easy to see that such an indexing exists.) Then let

$$
C=\mathcal{C}(\mathcal{E})=\left(\left(E_{1} * E_{2}\right) * \cdots\right) * E_{k} .
$$

Now, we are going to show that this definition is independent from the indexing of $\Sigma=\left\{S_{1}, \ldots, S_{k}\right\}$. There are four cases depending on how many times the position $(i, j)$ is in the sets $\left\{S_{1}, \ldots, S_{k}\right\}$ :

(i) If there is exactly one $l$ such that $(i, j) \in S_{l}$, then

$$
C(i, j)=e_{i j}^{(l)}
$$

where $e_{i j}^{(l)}$ denotes the value of $E_{l}$ in the position $(i, j)$.

(ii) If there are exactly two different $l_{1}$ and $l_{2}$ such that $(i, j) \in S_{l_{1}}$ and $(i, j) \in S_{l_{2}}$, then $C(i, j)$ is undefined.

(iii) If there are exactly three different $l_{1}, l_{2}$, and $l_{3}$ such that $(i, j) \in S_{l_{1}} \cap$ $S_{l_{2}} \cap S_{l_{3}}$, then two of the elementary switching patterns (say, $E_{l_{1}}$ and $E_{l_{3}}$ ) has the same value in the position $(i, j)$ and the other one $\left(E_{l_{2}}\right)$ has a different value here, i.e., $e_{i j}^{\left(l_{1}\right)}=e_{i j}^{\left(l_{3}\right)}=1-e_{i j}^{\left(l_{2}\right)}$. In this case

$$
C(i, j)=e_{i j}^{\left(l_{1}\right)}=e_{i j}^{\left(l_{3}\right)} \text {. }
$$

(iv) If there are exactly four different $l_{1}, l_{2}, l_{3}$, and $l_{4}$ such that $(i, j) \in S_{l_{1}} \cap$ $S_{l_{2}} \cap S_{l_{3}} \cap S_{l_{4}}$, then $C(i, j)$ is undefined.

That is, the value of $C(i, j)$ can be decided simply on the base of the parity of the number of discrete squares of $\Sigma$ covering $(i, j)$ (independently from the indexing of $\Sigma$ ). Accordingly, if $\mathcal{E}$ is the set of $2 \mathrm{D}$ elementary switching patterns of the same type on a strongly side-connected set, then we can $\operatorname{simply}$ write $\mathcal{C}(\mathcal{E})$ to denote the compositions of the elements of $\mathcal{E}$. As an example, see Fig. 5. 


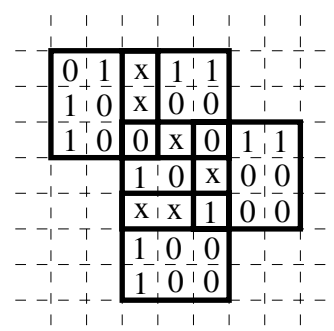

Fig. 5. The composition of the elementary switching patterns $E_{(2,2)}^{(0)}, E_{(2,4)}^{(0)}$, $E_{(4,4)}^{(0)}, E_{(4,6)}^{(0)}$, and $E_{(6,4)}^{(0)}$ ('x' denotes 'don't care' position)

Lemma 2. Let $\mathcal{E}$ be a set of elementary switching patterns of the same type on a strongly side-connected set of $3 \times 3$ squares. Then $\mathcal{C}(\mathcal{E})$ is a switching pattern.

Proof. The proof is given in [4].

Henceforth, the switching patterns constructed from elementary switching patterns of the same type by composition are called composite switching patterns. We say that the composite switching pattern has 0-type/1-type if it is the composition of 0-type/1-type elementary switching patterns. For example, (15)-(17) and Fig. 5 show composite switching patterns of types 0, 0, 1, 1, and 0.

\subsection{Composition of Corner-Connected Components}

Lemma 3. Let $\Sigma^{(0)}$ and $\Sigma^{(1)}$ be corner-connected components. Let $C^{(0)}$ and $C^{(1)}$ be 0-type and 1-type composite switching patterns on $\Sigma^{(0)}$ and on $\Sigma^{(1)}$, respectively. Then $C^{(0)} * C^{(1)}$ is a switching pattern.

Proof. The proof is given in [4].

Let $\sigma$ be a corner-connected set of strongly side-connected components $\Sigma^{(1)}$, $\ldots, \Sigma^{(k)}(k \geq 1)$. Let $C^{(1)}, \ldots, C^{(k)}$ be 0 - or 1 -type composite switching patterns on $\Sigma^{(l)}, \ldots, \Sigma^{(k)}$, respectively. Let us suppose also that if $\Sigma^{(l)}$ and $\Sigma^{\left(l^{\prime}\right)}$ are corner-connected then $C^{(l)}$ and $C^{\left(l^{\prime}\right)}$ have different type. In this case we say that $\gamma=\left\{C^{(1)}, \ldots, C^{(k)}\right\}$ is a set of alternatively corner-connected components on $\sigma$.

The composition of $\gamma$ on $\sigma$, denoted by $\mathcal{C}(\gamma)$, is defined as follows. If $k=1$ then $\mathcal{C}(\gamma)=C^{(1)}$. If $k>1$ then let us suppose that $\Sigma^{(1)}, \ldots, \Sigma^{(k)}$ are indexed such that for each $l(l<k)\left\{\Sigma^{(1)}, \ldots, \Sigma^{(k)}\right\}$ is a corner-connected set and one of its elements is corner-connected with $\Sigma^{(l+1)}$. (It is easy to see that such an indexing exists.) Then let

$$
C=\mathcal{C}(\gamma)=\left(\left(C^{(1)} * C^{(2)} * \cdots\right) * C^{(k)} .\right.
$$


It is easy to show that this definition is independent from the indexing of $\sigma$, because $\operatorname{dom}(C)=\cup_{l} \Sigma^{(l)} \backslash \cup_{l, l^{\prime}}\left(\Sigma^{(l)} \cap \Sigma^{\left(l^{\circ}\right)}\right)$ and $C(i, j)=C^{(l)}(i, j)$, where $l \in\{1, \ldots, k\}$ is the only index such that $(i, j) \in \Sigma^{(l)}$. Henceforth, we may simply write $\mathcal{C}(\gamma)$ to denote the composition of the elements of $\gamma$. As an example, see Fig. 6 .

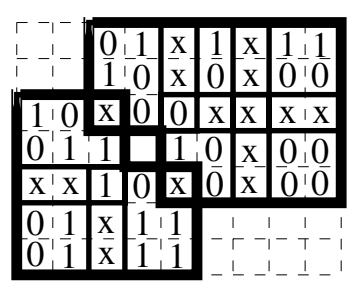

Fig. 6. Composition of two alternatively corner-connected components (the two components are indicated with thick lines)

Theorem 1. Let $\gamma=\left\{C^{(1)}, \ldots, C^{(k)}\right\}(k \geq 1)$ be a set of alternatively cornerconnected components. Then $\mathcal{C}(\gamma)$ is a switching pattern.

Proof. It follows from Lemma 3 directly.

Theorem 2. If a binary matrix has a switching pattern $C$ then there is a finite number of sets $\gamma_{1}, \ldots, \gamma_{l}(l>1)$ of alternatively corner-connected components such that $C$ is the composition of $\mathcal{C}\left(\gamma_{1}\right), \ldots, \mathcal{C}\left(\gamma_{l}\right)$.

Proof. The proof is given in [4].

Therefore, on the base of Theorems 1 and 2, it is proven that the 2D switching patterns are just the patterns created as the composition of alternatively cornerconnected components.

Finally, two consequences are mentioned (c.f. [6]):

(i) A binary matrix is uniquely determined by its absorbed row and column sums if and only if it has no sub-pattern created by composition from a set of alternatively corner-connected components.

(ii) If $A$ and $B$ are different binary matrices with the same absorbed row and column sums then $A$ is transformable into $B$ by a finte number of switchings of switching patterns created by compositions from sets of alternatively corner-connected components.

\section{Acknowledgements}

The authors thank to the reviewer to point out that one of the lemmas in the previous form of the paper was not correct. A part of this research was done while the first author visited the Laboratoire d'Informatique Algorithmique: Fondements et Applications, Université Paris 7 Denis-Diderot, Paris, France. Special 
thanks to Prof. Gabor T. Herman (Temple University, Philadelphia) and Laurent Vuillon (LIAFA, Université Paris 7) for their valuable comments and discussions. This work was supported by the grants FKFP 0908/1997 and OTKA T 032241, and MTA-NSF 123 joint grant supplement to NSF grant DMS 9612077 (Aspects of Discrete Tomography).

\section{References}

1. Brualdi, R. A.: Matrices of zeros and ones with fixed row and column sums. Linear Algebra and Its Applications 33 (1980) 159-231 139

2. Herman, G. T., Kuba, A. (Eds.): Discrete Tomography: Foundations, Algorithms and Applications. Birkhäuser, Boston (1999) 148

3. Kuba, A., Herman, G. T.: Discrete tomography: A historical overview. In [2] (1999) 3-34 139

4. Kuba, A., Nivat, M.: Reconstruction of discrete sets from projections in the case of absorption. Technical Reports, Institute of Informatics, University of Szeged (2000) 139, 141, 146, 147

5. Lothaire, M.: Combinatorics on Words. Cambridge University Press, Cambridge (1997) 138

6. Ryser, H. J.: Combinatorial properties of matrices of zeros and ones. Canad. J. Math. 9 (1957) 371-377 147 